\title{
SCIENZA PER CONVENZIONE?
}

\author{
LEOPOLDO POGGIALI (*)
}

SunTO. - In ambito scientifico il Convenzionalismo, nelle sue forme più o meno forti, sostiene che, a molteplici livelli e per molteplici motivi, nelle teorie siano presenti caratteri di convenzionalità. Si esporranno brevemente alcuni rilevanti argomenti di dibattito sul grado di convenzionalità della scienza individuando un convenzionalismo linguistico da affiancarsi ad un convenzionalismo metodologico e si presenteranno i punti di interazione e commistione tra i due. Si discuterà inoltre di come l'eventuale convenzionalità dell'impianto teorico implichi la convenzionalità degli aspetti della matematica ed dell'ontologia della teoria adottata.

$* * *$

ABSTRACT. - Scientific Conventionalism claims that conventional traits stands in theories in many forms and for many reasons. Relevant arguments about conventionalities in science will briefly be discussed, pointing out a Linguistic Conventionalism and a Methodological Conventionalism. We will show the common ground between the two and how they interact. Eventually we will highlight how conventionality of theoretical framework implies conventionality of the mathematics and the ontology adopted.

\section{LA NASCITA DEL DIBATTITO MODERNO}

Il Convenzionalismo si declina in ambito scientifico con molteplici sfaccettature, dando vita ad un dibattito tuttora vivo. Le origini delle riflessioni convenzionaliste in epoca moderna sono da ricercare nella nascita e nello sviluppo delle geometrie non euclidee, grazie alle quali trovano gli iniziali argomenti di dibattito. Nel corso del XX secolo il Convenzionalismo amplia i suoi orizzonti di intervento fino a comprendere da un lato la fondazione della logica e dall'altro le problematiche

(*) Università degli Studi di Pavia, Italia. E-mail: poggialileopoldo@gmail.com 
strettamente più epistemologiche relative alla struttura linguistica delle teorie, al problema della sottodeterminazione e dell'olismo della conferma. L'orizzonte all'interno del quale, quindi, muoversi per dare un'immagine seppur parziale delle riflessioni su questi temi è incredibilmente ampio ed è necessario procedere tramite un campionamento esemplare degli autori che possono essere rilevanti. Infatti innumerevoli celebri personaggi nell'ambito dell'Epistemologia si sono misurati con riflessioni di questo tipo sia sostenendo sia attaccando posizioni convenzionaliste ma in entrambi i casi dimostrando come il dibattito si sia mantenuto vivo fino ai giorni nostri. Si vuole qui dare una breve introduzione alle posizioni convenzionaliste di maggiore interesse nell'ambito del tema della conferenza.

Storicamente si riconosce la nascita del Convenzionalismo Scientifico con una serie di riflessioni proposte da Henrì Poincaré (1854-1912), sviluppatesi a partire dagli ultimi anni del decennio '90 del XIX secolo fino alla pubblicazione ne La Scienza e l'Ipotesi [1]. Poincaré propone un noto caso di studio che prende il suo nome: la Sfera di Poincaré. Questo esperimento mentale consiste in un mondo limitato racchiuso in una sfera finita dove sia presente un gradiente di temperatura radiale con andamento decrescente dal centro verso l'esterno descritto dall'espressione $T(r)=T_{0}\left(R^{2}-r^{2}\right)$, dove $T$ sta per la temperatura, $T_{0}$ per la temperatura al centro della sfera, $R$ per il raggio della sfera e $r$ per la coordinata radiale. Conseguentemente a questo gradiente di temperatura variano tutte le lunghezze fisiche, metri compresi, e l'indice di rifrazione della luce in maniera tale che ad un osservatore interno alla sfera il mondo apparirà illimitato e validamente descritto da una geometria ellittica. Questo esperimento mentale si pone come proposta di caso di studio patologico sulla scia di alcuni interventi pubblici tenuti nel 1870, e successivamente pubblicati nel 1876 [2], da Hermann L.F. von Helmholtz (1821-1894) a proposito delle possibilità offerte dalle geometrie non euclidee illustrate attraverso la descrizione di una realtà molto simile all'oggetto del noto libro Flatlandia di Abbott [1884]. Helmholtz mostrava come in un ipotetico mondo bidimensionale che si sviluppi sulla superficie di una sfera ci si trovi di fronte ad una possibile descrizione euclidea dello spazio su una piccola scala, ovvero quando le distanze misurate sono trascurabili al confronto con il raggio della sfera su cui questa realtà si sviluppa, mentre la "vera" geometria da utilizzare sarebbe dovuta essere non euclidea. Helmholtz giungeva così a posizioni oggi chiamate col nome di 
Empirismo Geometrico, affermando che la conoscenza della corretta geometria da utilizzare per descrivere il mondo fosse da raggiungere tramite dati empirici sempre più completi, nel suo caso misure di lunghezze non più trascurabili al confronto col raggio della sfera.

Nel caso della sfera di Poincaré, al contrario delle conclusioni tratte da Helmholtz, mentre per un osservatore interno alla sfera la scelta della geometria assolutamente più naturale per descrivere la realtà sarà quella ellittica, un osservatore esterno sarà d'altra parte consapevole della possibilità di descrivere il mondo della sfera sia con una geometria ellittica sia con una geometria euclidea, con l'aggiunta di opportune ipotesi sul gradiente di temperatura e su tutte le conseguenze illustrate sopra. Continua Poincaré, non sono possibili esperienze che mostrino quale è la geometria corretta da utilizzare perché entrambe riescono a descrivere completamente e correttamente i fenomeni ed anche gli argomenti metateorici comunemente diffusi, come per esempio la semplicità della trattazione, sono di scarso aiuto perché se da un lato abbiamo la semplicità della geometria euclidea complicata da una serie di ipotesi da assumere sul comportamento delle lunghezze e degli indici di rifrazione, dall'altra abbiamo la complessità della geometria non euclidea. La scelta di quale geometria utilizzare non può che essere una scelta convenzionale. Le conseguenze di ciò riguardano direttamente lo statuto della geometria che fino a quel momento, sulla scorta delle dominanti posizioni kantiane a riguardo, veniva considerata un a priori ; creando un'alternativa sia all'apriorismo sia all'empirismo à la Helmholtz.

\section{SOTTODERMINAZIONE E TESI DI DUHEM-QUINE}

La prima immediata applicazione di una posizione convenzionalista nel senso di Poincaré riguarda la soluzione al problema della sottodeterminazione delle teorie. In estrema sintesi e nel caso proposto anche dalla Sfera di Poincaré, questo problema consiste nell'impasse a cui ci si trova di fronte quando due teorie, basate su ipotesi differenti e potenzialmente con differenti ontologie, sono entrambe empiricamente adeguate. Risolvere questo stallo sta nello scegliere una teoria o l'altra sulla base di argomenti metateorici di carattere convenzionale quali semplicità della teoria, eleganza formale, maggiori punti di contatto con altre teorie e via dicendo. Al riguardo, Poincaré stesso sostiene che, seb- 
bene nell'immediato sia necessario prendere una scelta convenzionale, esistano scelte più convenienti di altre quando continue e recalcitranti esperienze rendano una delle teorie meno efficiente. Per questo sostiene anche che queste convenzioni siano sì presenti e in parte protagoniste del meccanismo decisionale, ma non siano completamente arbitrarie dato che, soprattutto in Fisica, l'attenzione al dato sperimentale deve sempre essere tenuta in primissima considerazione. Per approfondimenti sul Convenzionalismo in Poincaré e sulle sue influenze presso gli empiristi logici si veda Ref.[3], per un'esposizione dello scenario filosofico di sviluppo del Convenzionalismo anche nei confronti dell'Empirismo Logico si veda Ref.[4].

Legate al problema della sottodeterminazione delle teorie sono la questione dell'olismo della conferma e la tesi di Duhem-Quine poiché per entrambe sono state proposte chiavi di lettura convenzionaliste sulla falsariga del problema sopra esposto della sottodeterminazione. La questione prende il nome da Pierre M.M. Duhem (1861-1916), che per primo formulò l'argomento ne La théorie physique: son objet, et sa structure [5] nell'ambito delle teorie fisiche e come critica al concetto di experimentum crucis, introdotto da Francis Bacon (1561-1626) nel suo Novum Organon [6], e da Willard Van Orman Quine (1908-2000) che ne dette una formulazione più generale nel suo celebre Two Dogmas of Empiricism [7]. L'experimentum crucis consiste in una esperienza empirica il cui outcome sia un'indicazione definitiva su quale scegliere tra due ipotesi in contrasto, uno dei casi di questo tipo entrato nella storia è l'esperimento di Léon Foucault a proposito della natura corpuscolare od ondulatoria della luce. Tradizionalmente l'experimentum crucis era considerato la tappa finale nella scelta tra ipotesi contrapposte poiché dava un risultato chiaro direttamente dipendente dalle ipotesi in dubbio, Duhem però sostiene che nessuna predizione osservativa è derivata da un'unica ipotesi in quanto gli elementi di una teoria sono interconnessi. Infatti, se una certa predizione osservativa viene derivata da un insieme di ipotesi (cosa che avviene regolarmente, soprattutto con il progressivo aumento della sofisticazione degli apparati di misura) e questa previsione viene smentita, allora la sola cosa che se ne può dedurre è che l'insieme delle ipotesi deve essere negato, il che logicamente equivale a negare una delle ipotesi, la quale potrebbe riguardare il funzionamento dell'apparato sperimentale così come l'esistenza o meno di entità teoriche postulate precedentemente. Tuttavia, in mancanza di altri dati, non si può decidere quale delle ipotesi sia quella 
falsa. C'è quindi una libertà nel riformulare la teoria, scegliendo quale ipotesi si vuole che sia falsificata o quale ipotesi aggiungere alla nostra teoria affinché eventuali dati sperimentali anomali possano rientrare come proposizione osservativa regolare ex post.

L'argomento ben si posiziona d'altronde all'interno della concezione di Duhem della teoria fisica come economy of thought, un insieme di proposizioni matematiche derivate da pochi principi con lo scopo di rappresentare semplicemente e in maniera completa l'insieme di quello che chiamava "leggi sperimentali", ovvero gli andamenti fenomenologici rilevati dagli esperimenti, ma senza nessun interesse verso la Natura o le Entità tout court. Concezione, questa, che si avvicina in alcuni aspetti alle posizioni di alcuni esponenti dell'Empirismo Logico, di cui parleremo in seguito. Per Quine invece questo modo di procedere è proprio non solo dello sviluppo della scienza ma in generale di tutta la conoscenza umana. Infatti, afferma "Tutte le nostre cosiddette conoscenze [. . . ] sono un edificio fatto dall'uomo che tocca l'esperienza solo lungo i suoi margini. [. . . ] L'intero campo è determinato dai suoi punti limite, cioè l'esperienza, in modo così vago che rimane sempre una notevole libertà di scelta per decidere quali siano le proposizioni di cui si debba dare una nuova valutazione alla luce di una certa particolare esperienza contraria"[7], originale inglese.

In entrambe queste formulazioni, l'argomento di Duhem-Quine ben si presta ad una chiave di lettura convenzionalista: la scelta arbitraria di quale parte dell'edificio teorico o di tutto l'edificio conoscitivo sia da cambiare e riadattare è presa alla luce di motivi di natura convenzionale, nel senso stretto di scelte condivise dalla comunità di praticanti del determinato settore in cui la questione viene posta, siano comunità scientifiche, comunità di parlanti un certo linguaggio o altri esempi su questa scia. Per un approfondimento sulle differenze tra il convenzionalismo di Poincaré e di Duhem si veda Ref.[8].

\section{IL CONVENZIONALISMO LOGICO}

Tornando al caso della Sfera di Poincaré, un'altra questione, appena accenata sopra, che veniva posta riguardava lo status della geometria. Se infatti la scelta della geometria è convenzionale, se la geometria è fondata sulla matematica e quest'ultima, anche se con qualche riserva, sulla logica, allora si può pensare di far risalire il fondamento 
convenzionalista della geometria direttamente alla logica, ovvero intraprendere il programma del cosiddetto Convenzionalismo Logico. Gli sforzi in questo senso sono diretti nel sostenere la tesi per cui qualunque proposizione $p$ logicamente vera è resa tale da convenzioni linguistiche. A seconda che queste convenzioni linguistiche abbiano la forma esplicita o implicita di regole sull'utilizzo di $p$, si parla di convenzionalismo logico esplicito o implicito.

La prima forma venne efficacemente attaccata da Quine nel noto Truth by convention, si veda Ref.[9], nel quale mostra come sostenere che le convenzioni linguistiche debbano avere forma esplicita di regole a proposito di $p$ porta inevitabilmente ad un ragionamento circolare. Partendo dalla constatazione che le definizioni sono convenzioni su abbreviazioni e che la scienza è avanzata tramite l'introduzione di definizioni, Quine afferma che le definizioni non possano far altro che mantenere e trasmettere la verità e mai potranno fondarla, come invece serve che facciano le regole convenzionali esplicite, nella forma linguistica di un "accordo" a proposito di $p$. Per questo, se la logica è da fondarsi su queste regole esplicite per poi essere dedotta da queste, evidentemente si sta presupponendo la logica alla sua stessa derivazione e andando incontro ad un argomento circolare espresso, invece che dal suggestivo logic is true by convention, dallo slogan logic is true by convention plus logic, che evidentemente perde tutto l'appeal a cui poteva sperare.

Unico modo di salvare la tesi convenzionalista logica è sostenere di adottare le convenzioni linguistiche tramite il comportamento prima ancora di formularle in maniera esplicita nel linguaggio utilizzato. Queste sono le posizioni del Convenzionalismo Logico Implicito, per le quali la formulazione verbale delle regole linguistiche non è più prerequisito per l'adozioni delle stesse e una eventuale esposizione esplicita rappresenta solo uno degli utilizzi di un linguaggio completo: le regole che governano l'uso dei connettivi logici spiegano a pieno la verità di proposizioni logicamente vere nel linguaggio in questione. L'argomento di Quine è disarmato nei confronti di questo Convenzionalismo Logico Implicito ma Quine stesso, presa in considerazione questa formulazione, semplicemente sostiene che le regole adottate implicitamente come descritto sopra non sarebbero altro che verità banali senza nessun valore fondativo. Negli anni successivi alla pubblicazione di Truth by Convention però, con merito anche dei lavori di Noam Chomsky (1928 - vivente) ed altri, molti recenti studi di filosofia della mente e del linguaggio mostrano come ci sia modo di distinguere tra le verità logiche 
presupposte e le mere ovvietà, si vedano Refs.[11], [12] e [13], dando nuova linfa vitale a questo genere di posizioni, molto vicine del resto alla forma di Inferenzialismo Logico perseguito dagli Empiristi Logici, primo tra tutti Rudolf Carnap (1891-1970). Per un'analisi dell'inefficacia dell'argomento di Quine si veda Ref.[10].

\section{CONCEZIONE SINTATTICO-LINGUISTICA DELLE TEORIE}

Proprio nel pensiero di Carnap, ma in generale di molti esponenti dell'Empirismo Logico come vedremo in seguito, si trovano altre posizioni convenzionaliste in senso empistemologico e scientifico. Nel suo Logische Syntax der Sprache, Ref.[14], Carnap offre una sistematizzazione della struttura convenzionale delle forme linguistiche nell'ambito delle teorie scientifiche. Introducendo la differenza tra quelle che chiama L-proposizioni e P-proposizioni, ovvero, sostanzialmente, proposizioni analitiche e non, propone una visione delle teorie come sistemi di proposizioni dove elementi convenzionali spaziano l'impianto concettuale all'interno del quale i fatti possono essere espressi e spiegati, in qualche maniera vicino a quel che sopra abbiamo detto di Duhem.

Di nuovo in sintesi, Carnap sostiene che occorra distinguere tra le proposizioni formulate all'interno di un linguaggio e quelle che invece si riferiscono alle proposizioni del linguaggio. Questo secondo tipo di proposizioni costituisce il metalinguaggio, il linguaggio che ha come riferimento un altro linguaggio. L'obiettivo del lavoro sopra citato consiste nel costruire la teoria formale delle forme linguistiche: tale è la sintassi logica del linguaggio che stabilisce le regole in base alle quali costruire le strutture linguistiche. In più, la teoria che Carnap tratteggia consiste di regole puramente formali poiché non fanno riferimento al significato delle proposizioni di cui trattano o della loro combinazione: è un insieme di regole che determinano se un enunciato è correttamente costruito e che descrivono come possa essere derivato da un altro, rispettivamente sono quindi regole di formazione e di trasformazione. Grazie a queste regole si può passare dai P-primitivi, gli oggetti teorici introdotti nella teoria fisica, alle P-proposizioni, affermazioni riguardo questi oggetti, ed infine agli enunciati protocollari, enunciati osservativi dotati di senso, ossia, nell'accezione dell'Empirismo Logico, verificabili. Ci si trova quindi di fronte ad un costrutto di proposizioni matematico-formali senza un contenuto semantico che rappresentano degli ausiliari formali all'interno dell'insieme dei 
quali è possibile definire $\mathrm{i}$ concetti di interesse come per esempio quelli di giustificazione, di correttezza o di verificabilità e via dicendo, si veda a proposito anche Ref.[15].

Queste regole di formazione e trasformazione dei segni possono quindi essere fissate arbitrariamente perché sono esse che definiscono il linguaggio al quale si devono applicare. L'aspetto di convenzionalità in questo costrutto ha evidentemente una duplice natura: da un canto possiamo scegliere convenzionalmente il linguaggio e dall'altro possiamo scegliere arbitrariamente anche le regole sintattiche di questo linguaggio. Per ulteriori considerazioni sul Convenzionalismo in Carnap si veda Ref.[16] e per approfondire il dibattito tra Carnap e Quine sui temi del Convenzionalismo Logico si veda Ref.[17]. Questi framework linguistici sono da scegliere opportunamente perché si rivelino efficaci nella pratica scientifica ma non sono mai completamente determinati empiricamente, anche e soprattutto perché sono privi di contenuto semantico. Questo ci collega all'ultima parte dell'intervento e di questa sua stesura riassuntiva: quello che chiameremo Convenzionalismo Metodologico per distinguerlo da quello che chiameremo Convenzionalismo Linguistico.

Le considerazione fatte finora sono strettamente legate all'aspetto linguistico delle teorie fisiche, e scientifiche in generale: a partire dalla scelta del formalismo con cui descrivere i fenomeni, il problema della sottodeterminazione, fino alla struttura sintattica convenzionale delle teorie passando dal Convenzionalismo Logico. Si può però individuare un altro tipo di applicazioni di argomenti convenzionalisti che riguardano in primo luogo l'applicazione della teoria, quindi in prima approssimazione il metodo con cui si utilizza quest'ultima, e, solo in maniera indiretta, l'aspetto linguistico, il quale però, alla luce della concezione della teoria scientifica che abbiamo visto essere propria di molti personaggi di rilievo, tra i citati Duhem e Carnap, come insieme linguistico di proposizioni, resta sempre presente anche se mimetizzato. Questo genere di argomenti non ha che fare con il valore di verità delle proposizioni o con la costruzione corretta di proposizioni, riguarda piuttosto la connessione tra le strutture matematiche e il contenuto empirico della teoria.

\section{IL CONVENZIONALISMO METODOLOGICO E I PRINCIPI CORDINATIVI}

Le riflessioni a cui facciamo riferimento in questo senso sono quelle di Hans Reichenbach (1891-1953), anch'egli come Carnap esponente 
dell'Empirismo Logico. Nel suo The Theory of Relativity and A Priori [20] Reichenbach parte da un'analisi dell'a priori kantiano distinguendone due accezioni: una di necessariamente vero, che, sostiene, ha perso di consistenza con l'avvento delle geometrie non euclidee e della teoria della relatività, ed una di costituente il concetto stesso degli oggetti ma non costituente dell'esperienza tout court. Anche alla luce di ciò che abbiamo detto sopra parlando della sintassi logica del linguaggio, quest'ultima accezione implica che questi a priori sono costitutivi della conoscenza scientifica ottenibile in un certo framework teorico. Reichenbach chiama questi elementi "principi coordinativi", ovvero quei principi che connettono le strutture matematiche (le formule logico-formali alle quali si riferisce Carnap) al contenuto empirico delle teorie, che, come sostiene con forza Reichenbach, dà una semplificata immagine del contenuto delle percezioni, troppo complesse e non riducibili al semplice contenuto delle equazioni di leggi matematiche, nel caso della Fisica. Si possono individuare tre diverse applicazioni dei principi coordinativi: le convenzioni associative, le descrizioni equivalenti e la convenzionalità delle congruenze.

\section{LE DESCRIZIONI EQUIVALENTI}

La questione delle descrizioni equivalenti riguarda il problema degli oggetti inosservati. Questo problema è strettamente collegato alla concezione di significato degli enunciati dettata dall'Empirismo Logico: il significato di un enunciato consiste nella sua modalità di verifica. Si consideri poi che gli enunciati protocollari, che rappresentano il contatto autentico della teoria con la realtà che si suppone essa descriva, sono enunciati intrinsecamente legati dalla procedura stessa di osservazione e conseguentemente agli oggetti che sono al centro di queste osservazioni, ovvero gli oggetti osservati. Reichebach sostiene però che avvenga un'automatica assunzione di similitudine tra gli oggetti inosservati e quelli osservati. Come abbiamo detto la teoria contiene all'interno del suo dominio gli oggetti osservati: è a loro proposito che la teoria può produrre previsioni ed è a causa loro se la teoria viene falsificata; nella pratica scientifica però si mette in pratica una regola di estensione del linguaggio nel momento in cui si include nel dominio oltre agli oggetti osservati, anche tutti i loro presupposti simili. In questo modo assumiamo che gli oggetti inosservati siano governati dalle stesse leggi verificate per gli oggetti osservati. 
Questa scelta, continua Reichenbach, ha come primo scopo la semplificazione linguistica della teoria, e quindi come accennato rientra in una manifestazione di Convenzionalismo Linguistico, ma riguarda di fatto la modalità di utilizzo della teoria. La scelta di estendere il linguaggio nel modo sopra illustrato è una scelta arbitraria e convenzionale presa in un'ottica di semplificazione teorica mentre è comunque possibile scegliere altrimenti trovando altri argomenti metateorici, e per questo non meno stringenti della semplicità della teoria, per motivare la scelta alternativa. In ogni modo, c'è certamente una necessità di introdurre convenzione a proposito degli oggetti inosservati per il fatto che è logicamente impossibile osservare un oggetto inosservato, per cui è logicamente impossibile che questi rientrino a pieno titolo nel dominio della teoria.

\section{CONVENZIONALITÀ DELLE CONGRUENZE}

Reichenbach parla poi di convenzionalità delle congruenze partendo dal problema del confronto. Questo problema veniva solitamente posto da Reichenbach all'inizio del suo corso agli studenti riguardo la modalità di verifica che un oggetto fosse più grande di un altro, entrambi all'interno dell'aula dove si teneva la lezione ma distanti l'uno d'altro. Alle risposte degli studenti veniva mostrato come di fatto si fosse di nuovo di fronte ad una impossibilità logica della verifica di quell'affermazione dato che, in ogni modo si ponesse la soluzione, si doveva comunque assumere convenzionalmente il mantenimento delle lunghezze durante la fase di moto, fosse la lunghezza di un eventuale metro con il quale si erano misurati gli oggetti o fosse la lunghezza degli oggetti stessi portati l'uno di fianco all'altro. Tutto ciò che è possibile sapere è che gli oggetti sono uno più grande dell'altro nel posto nel quale vengono comparati. Lo stesso si può argomentare per la risposta alla domanda su quale intervallo di tempo tra coppie di eventi fosse più lungo. Con le parole dello stesso Reichebach: "We cannot carry back the later time interval and place it next to the earlier one ([21], pg. 116)".

In generale la convenzionalità delle congruenze si applica a tutti i tipi di congruenze, perfino alla questione dell'identità nel tempo dei sistemi fisici rispetto a loro stessi, evidentissima nel formalismo delle Teorie Operazionali. Come detto, data l'impossibilità logica della veri- 
fica di tali congruenze, queste prendono inevitabilmente un carattere di convenzionalità che è sì indipendente dalle esperienze, ma che ne determina lo svolgimento e determina il sistema concettuale che si sviluppa con la scienza, di nuovo con le parole dello stesso Reichenbach: "[ . . . ] congruence is not a matter of knowledge but a matter of definition ([21], pg. 116)”.

\section{CONVENZIONI ASSOCIATIVE}

Infine, le convenzioni associative riguardano in maniera esplicita il problema del collegamento tra entità teoriche e dati sperimentali. Come abbiamo detto, nelle teorie fisiche raffigurate come teorie sintattico-linguistiche e soprattutto nelle teorie assiomatizzate, quale è la Teoria Quantistica [22], la sintassi della teoria è distinta dalla semantica. Niente all'interno della teoria può far riferimento in maniera autonoma ad entità esterne alla teoria ma, come di nuovo sostiene Reichenbach, non si deve dimenticare che gli oggetti della teoria sono entità astratte che descrivono oggetti e eventi del mondo fisico ma non ne condividono lo statuto: sono semplificate astrazioni matematico-formali. Da qui la necessità di determinare il riferimento dei termini teorici.

Si prenda come esempio la Teoria Quantistica assiomatizzata citata sopra. E`stato mostrato, si veda di nuovo Ref.[22], come, all'interno del formalismo delle Teorie Operazionali, partendo da sei principi informazionali si possa ricostruire la teoria con la sua sovrastruttura matematica comunemente utilizzata. Questi principi però, come in generale gli assiomi di una qualunque teoria assiomatizzata, fanno riferimento ai termini primitivi della teoria, dei quali rappresentano le definizioni implicite. I termini primitivi d'altro canto sono astrazioni formali, i P-primitivi di cui parla Carnap; sono pertanto elementi della sintassi della teoria e, rigorosamente, non hanno niente a che spartire con gli oggetti reali del mondo che la teoria vuole descrivere. Il collegamento tra questi primitivi astratto-formali e i loro corrispondenti fisici non può essere concepito in termini dei principi della teoria dal momento che essi si riferiscono, come abbiamo detto, solo ai primitivi stessi. Ecco la necessità di un contributo esterno alla teoria per mettere in rapporto queste due realtà. Si collegano i due insiemi tramite regole che si scelgono in maniera mai del tutto determinata empiricamente, come puntualizza anche Carnap, dato che anche l'interpretazione delle esperien- 
ze empiriche avviene all'interno della teoria stessa e quindi già in termini di sintassi della teoria. Le regole di collegamento sono scelte convenzionalmente e spesso neanche esplicitate nel linguaggio della teoria né in quello naturale.

Questa scelta non sarebbe oggetto di controversia se la nostra libertà fosse di scegliere tra differenti formulazioni della stessa teoria soltanto od al massimo di teorie equivalenti; purtroppo la questione diventa critica quando non è possibile una corrispondenza sistematica tra le descrizioni, come nel caso di termini teorici imparagonabili tra teorie incommensurabili. Dall'altro lato, la necessità di questo genere di principi coordinativi si mostra innanzitutto, come abbiamo visto, come necessità logica e conseguentemente come richiesta per la percezione della struttura del mondo fisico, anche se la scelta di quale principio non è determinata unicamente dall'esperienza. Per una trattazione riassuntiva del Convenzionalismo di Reichenbach, in particolare di queste posizioni e argomenti sui principi coordinativi, si vedano Reff.[18] e [19].

In conclusione possiamo dire che i principi coordinativi, così come introdotti da Reichenbach, assumono un duplice ruolo all'interno dell'edificio della conoscienza scientifica. Da un lato essi sono indipendenti dalle osservazioni e, come abbiamo più volte detto, di natura convenzionale; dall'altro però non c'è indipendenza delle osservazioni da questi: essi determinano in maniera non trascurabile il sistema concettuale che sviluppa la scienza, andando ad incidere sull'interpretazione e sul significato delle osservazioni all'interno della teoria stessa. Si apre così la strada a considerazioni di theory ladenness che renderebbero ancora più manifesto il ruolo cruciale delle convenzioni nella scienza, ma che non abbiamo modo di approfondire in questa sede, e in special modo, alla luce di tutte queste considerazioni fatte, nell'ambito di molti argomenti propri del dibattito sul Realismo Scientifico, il quale non si può pensare rimanga immune ad una critica convenzionalista.

\section{BIBLIOGRAFIA}

[1] Poncaré H. La Science et l'Hypothèse. Parigi: Ernest Flammarion E diteur, 1902.

[2] Helmholtz H. "The Origin and Meaning of Geometrical Axioms”. In: Mind 1.3 (1876), pp. 301-321.

[3] Friedman M. "Poincaré's conventionalism and the logical positivists". In: Foundations of Science 1.2 (June 1995), pp. 299-314. 
[4] Haller R. "Conventionalism and it's impact on logical empiricism". In: Philosophia Scientiae 3.2 (), pp. 95-108.

[5] Duhem P.M.M. La théorie physique: son objet, et sa structure. Bibliothèque de philosophie expérimentale. Chevalier \& Rivière, 1906.

[6] Bacon F. Nuovo organo delle scienze di Francesco Bacone di Verulamio. D. Oliveri, 1839.

[7] Quine W.v.O. "Main Trends in Recent Philosophy: Two Dogmas of Empiricism”. In: The Philosophical Review 60.1 (1951), pp. 20-43.

[8] Ivanova M. "Conventionalism about what? Where Duhem and Poincaré Part Ways.”. In: Studies in History and Philosophy of Science Part A 54 (2015), pp. 8089.

[9] Quine W.v.O. "Truth by Convention”. In: Journal of Symbolic Logic. 1936, pp. 77-106.

[10] Warren J. "Revisiting Quine on Truth by Convention". In: Journal of Philosophical Logic 46.2 (2017), pp. 119-139.

[11] Chomsky N. and Halle M. The sound pattern of English. Studies in language. Harper \& Row, 1968.

[12] Everett D.L. Language: The Cultural Tool. Knopf Doubleday Publishing Group, 2012.

[13] Matthews P.H. “The linguistics wars”. In: Journal of Linguistics 32.2 (1996), $526 ? 527$.

[14] Carnap R. The Logical Syntax of Language. Open Court classics. Open Court, 2002.

[15] Carnap R. "Testability and Meaning”. In: Philosopby of Science 3.4 (1936), pp. 419-471.

[16] Yunez Naude N. "What is Carnap's Conventionalism after all?" In: Synthese 137.1 (Nov. 2003), pp. 261-272.

[17] Ebbs G. "Carnap and Quine on Truth by Convention". In: Mind 120.478 (2011), pp. 193-237.

[18] Beauregard L.A. "Reichenbach and Conventionalism". In: Synthese 34.3 (1977), pp. 265-280.

[19] Davidson L. "Necessary Principles of Perception: A Comparison of Kant and Reichenbach". In: The California Undergraduate Philosophy Review 1 (), pp. 8398.

[20] Reichenbach H. and Reichenbach M. The Theory of Relativity and a Priori Knowledge. University of California Press, 1965.

[21] Reichenbach H. The Philosophy of Space and Time. Dover Books on Physics. Dover Publications, 2012.

[22] D’Ariano G.M., Chiribella G., and Perinotti P. Quantum Theory from First Principles: An Informational Approach. Cambridge University Press, 2017. 